\title{
Article \\ Anthropometric Characteristics in Taiwanese Adults: Age and Gender Differences
}

\author{
Shih-Chang Chen ${ }^{1}$, Chaou-Wen Lin ${ }^{2}$, Po-Fu Lee ${ }^{1,3}{ }^{\mathbb{D}}$, Hui-Ling Chen ${ }^{4}$ and Chien-Chang Ho ${ }^{5,6,7, * \mathbb{D}}$ \\ 1 Department of Leisure Industry and Health Promotion, National Ilan University, Ilan County, \\ Yilan City 260, Taiwan; ncc5207@niu.edu.tw (S.-C.C.); f520184fred@yahoo.com.tw (P.-F.L.) \\ 2 Department of Marine Leisure and Tourism, Taipei University of Marine Technology, Taipei City 111, Taiwan; \\ mikelin319@mail.tumt.edu.tw \\ 3 Center of General Education, Taipei Medical University, Taipei City 110, Taiwan \\ 4 Graduate Institute of Educational Leadership and Development, Fu Jen Catholic University, \\ New Taipei City 242, Taiwan; 024145@mail.fju.edu.tw \\ 5 Department of Physical Education, Fu Jen Catholic University, New Taipei City 242, Taiwan \\ 6 Office of Physical Education, Fu Jen Catholic University, New Taipei City 242, Taiwan \\ 7 Research and Development Center for Physical Education, Health, and Information Technology, \\ Fu Jen Catholic University, New Taipei City 242, Taiwan \\ * Correspondence: 093703@mail.fju.edu.tw; Tel.: +886-2-2905-3386
}

Citation: Chen, S.-C.; Lin, C.-W.; Lee, P.-F.; Chen, H.-L.; Ho, C.-C. Anthropometric Characteristics in Taiwanese Adults: Age and Gender Differences. Int. J. Environ. Res. Public Health 2021, 18, 7712. https:// doi.org/10.3390/ijerph18147712

Academic Editor: Paul B. Tchounwou

Received: 2 June 2021

Accepted: 18 July 2021

Published: 20 July 2021

Publisher's Note: MDPI stays neutral with regard to jurisdictional claims in published maps and institutional affiliations.

Copyright: () 2021 by the authors. Licensee MDPI, Basel, Switzerland. This article is an open access article distributed under the terms and conditions of the Creative Commons Attribution (CC BY) license (https:// creativecommons.org/licenses/by/ $4.0 /)$.

\begin{abstract}
Population aging is creating critical issues in Taiwan, and adults are being forced to maintain productivity at work; in other words, they need to work longer. Therefore, their fitness and health warrant immediate attention. Although the association between health and anthropometric characteristics has been reported, few profiles on Taiwanese adults can be found. The purpose of this study was to provide a suitable reference on the anthropometric data of Taiwanese adults. We recruited 60,056 anthropometric measurements from a representative database. Significant differences were found in every measurement for each gender and age group. Statistically, our results indicated anthropometric differences in different ages. However, CVs showed that the dispersions are minor. This study presents a sufficient profile on Taiwanese adults from a representative database to practitioners and other potential users.
\end{abstract}

Keywords: obesity; BMI; WHR; Taiwan

\section{Introduction}

Taiwan has been reported as a super-aged society with a rapidly decreasing adult population [1]. In Taiwan, population aging is creating socioeconomic problems, and its impacts worldwide warrant immediate attention. For example, many countries have adopted pension systems to maintain sustainability [2-5]. Moreover, the increasing costs of public health services have also challenged society and its general productivity [6]. Thus, the health condition of adults has become more critical in Taiwan since they are responsible for the productivity of society.

Anthropometric parameters are commonly related to one's physical fitness [7], dietary status [8], lifestyle [9] and general health condition [10,11]. Although anthropometric parameters may be affected by genetic differences, environmental issues and sociocultural conditions, they can still provide significant information on clinical and epidemiological issues [12]. A recent study reported a longitudinal relationship between anthropometric parameters and stress and its tendency to cause overeating [13]. Over the past years, studies have focused on the associations between anthropometric parameters and potential employee selection [14], psychological issues [13], ergonomic product design [15,16] and touchscreen information system design [17]. Even though there are many more indicators that predict human health, anthropometric parameters, such as the body mass index (BMI) 
and waist-hip ratio (WHR), provide an easy and inexpensive measurement approach for communities [18].

According to the World Health Organization (WHO), anthropometric data should be collected regularly and provided as a standard reference for preliminary health monitoring [19]. These data are recommended to be shown in 10-year clusters and should be distinguished from gender differences [18-20]. However, to the best of our knowledge, anthropometric reference data have seldom been provided for adults in Taiwan, and previous studies have focused on adolescents [21] and the elderly [22].

In addition, these studies used a small sample for analysis. Increasing the quality of these data is essential. Even though these data may make a limited contribution to scientific development, sufficient reference data are worth to be established due to their practical value, especially for the adult population. Therefore, this study aimed to provide genderand age-specific characteristics of anthropometric parameters in Taiwanese adults by using a secondary database.

\section{Materials and Methods}

\subsection{Study Design and Participants}

Cross-sectional analysis was conducted by using the Taiwanese National Physical Fitness Survey database (NPFSIT). The NPFSIT is operated annually in order to survey the physical fitness level of citizens. The government supervises its procedures, data collection, data management and applications. The design, sampling protocols and data validation of the NPFSIT have been previously introduced [23-25], and de-identified data from the NPFSIT have been released for research. The data that this study has used were collected from 62,586 participants (29,685 men, 32,901 women) from October 2014 to March 2015. Convenient sampling was applied at 46 examination stations across 22 cities in Taiwan. The purpose and procedure of the NPFSIT were explained to the participants. All participants provided informed consent. The study design and analysis protocol were supervised by the Institutional Review Board of the Fu Jen Catholic University, Taiwan (FJU-IRB C108006).

\subsection{Data Collection}

Before data collection, some regional training seminars were conducted for the examiners to ensure the protocols and assessments could be correctly presented. All the examiners qualified for the training, as reported previously [26,27]. The study was conducted in three phases. The first was to complete the survey questionnaire. The items included sociodemographic characteristics, lifestyle and perceived health status. The second phase was to check each participant's resting heart rate and blood pressure for safety purposes. Participants whose systolic blood pressure exceeded $140 \mathrm{mmHg}$ or diastolic blood pressure exceeded $90 \mathrm{mmHg}$ or who reported heart disease, hypertension, chest pain, vertigo or musculoskeletal disorders were excluded. The last phase was an anthropometric variable assessment.

The sociodemographic items in the questionnaire were age, gender, education, monthly income and marital status. The lifestyle questions were related to smoking and betel nut chewing. A 5-point Likert scale measured the perceived health status by asking the participants whether they felt healthy. There were three levels of education (elementary school or lower, junior or senior high school and college or higher), monthly income (under 20,000 New Taiwan Dollar (d), 20,001 to 40,000 NTD, and above 40,001 NTD) and marital status (married, never married and divorced/separated/widowed). The participants were also asked whether they never, formerly or currently used cigarettes and/or betel nuts.

\subsection{Anthropometric Variable Assessment}

Anthropometrics were measured for weight, height, waist circumference (WC) and hip circumference (HC). The weight and height were measured by an automatic weight and height machine. The participants were asked to remove their shoes and heavy clothes and stand in a normal posture during measurement. Each WC and HC measurement was 
performed twice, and the mean value was calculated. The participants were asked to stand in a normal posture, breathe out and hold their breath for a second, and the WC between the lowest rib and the iliac crest was measured. Similarly, the HC was measured as the distance around the widest part of the buttocks (below the hip plates). The Taiwanese health administration recommends that men maintain their WC below $90 \mathrm{~cm}$ and women below $80 \mathrm{~cm}$ to avoid obesity [28].

The body mass index (BMI) and waist-hip ratio (WHR) were easily calculated. Based on the BMI, the participants were divided into four different groups: underweight, normal weight, overweight and obese. The cut-off points for four groups were 18.5, 24 and $27 \mathrm{~kg} / \mathrm{m}^{2}$, according to the Health Promotion Administration in Taiwan [29]. The WHR cut-off points were 0.9 for men and 0.85 for women [30].

\subsection{Statistical Analysis}

AS 9.4 software (SAS Institute, Cary, NC, USA) was used to analyze gender- and age-specific data. The statistical measurements included the mean, standard deviation and percentiles (5th, 25th, 50th, 75th, 85th, 90th and 95th). The age groups were 23-24, 25-34, $35-44,45-54$ and 55-64 years. The coefficient of variation (CV) was calculated (standard deviation (SD)/mean) for each anthropometric measurement to determine the dispersion. Means \pm standard deviation (SD) or frequency percentages were presented. Student's $t$-test and chi-square tests were performed for continuous and categorical variables, respectively. Tukey's post hoc test was used to compare the differences among the groups. The level of significance was set at $p<0.05$.

\section{Results}

Table 1 shows the demographics and anthropometrics of the 62,586 participants (29,685 men, 32,901 women). Both men and women were significantly different in each measurement (age, body weight, height, BMI, WC, HC, WHR, education, income level, marital status, self-reported health status, smoking status, betel nut chewing; $p<0.001$ ).

Tables 2-5 show the results of each anthropometric parameter (mean, standard deviation, CV and percentile) for both men and women, distributed by age. Significant differences were found in all mean values between genders $(p<0.05)$, and all mean values were higher in men than in women. In addition, mean values in both men and women were significantly different among ages $(p<0.05)$. In men, the mean WC and WHR in the eldest age group were higher than the younger age groups. In women, except for body weight, all the observed means in the eldest age group were significantly higher than in other groups $(p<0.05)$.

The median values (p50) of the body weight and BMI were slightly lower than the means in both men and women. Moreover, the median values of the WC, HC and WHR were slightly lower than the means in women. In general, the result indicated slightly skewed distributions with a wide dispersion for each measurement. Tukey's multiple comparisons test showed that in men, differences in the WHR were significant between all age groups; in addition, significant differences were found in the height, BMI and WC between the eldest (55-64 years) and youngest (23-24 years) age groups ( $p<0.05$; Tables 2 and 3). In contrast, the BMI, WC and WHR in women were significantly different in all age groups. In addition, the means of the body weight, height and HC were significantly different between the eldest and youngest age groups $(p<0.05$; Tables 4 and 5$)$. 
Table 1. Demographic and anthropometric characterization of the study population.

\begin{tabular}{|c|c|c|c|c|}
\hline Variables & $\begin{array}{c}\text { Total } \\
(N=62,586)\end{array}$ & $\begin{array}{c}\text { Men } \\
(n=29,685)\end{array}$ & $\begin{array}{c}\text { Women } \\
(n=32,901)\end{array}$ & $p$-Value \\
\hline Age (years) (\%) & & & & $<0.001$ * \\
\hline $23-24$ & 7.0 & 8.0 & 6.1 & \\
\hline $25-34$ & 25.4 & 28.2 & 23.0 & \\
\hline $35-44$ & 27.4 & 27.6 & 27.1 & \\
\hline $45-54$ & 21.9 & 20.9 & 23.0 & \\
\hline $55-64$ & 18.3 & 15.3 & 20.9 & \\
\hline Body weight (kg) & $64.4 \pm 12.2$ & $72.2 \pm 10.5$ & $57.4 \pm 8.8$ & $<0.001$ * \\
\hline Height $(\mathrm{cm})$ & $164.2 \pm 8.6$ & $170.7 \pm 6.3$ & $158.4 \pm 5.8$ & $<0.001$ * \\
\hline BMI $\left(\mathrm{kg} / \mathrm{m}^{2}\right)$ & $23.8 \pm 3.5$ & $24.8 \pm 3.3$ & $22.9 \pm 3.4$ & $<0.001$ * \\
\hline $\mathrm{WC}(\mathrm{cm})$ & $80.0 \pm 9.8$ & $84.5 \pm 8.7$ & $75.9 \pm 8.9$ & $<0.001$ * \\
\hline $\mathrm{HC}(\mathrm{cm})$ & $95.5 \pm 6.6$ & $96.9 \pm 6.2$ & $94.2 \pm 6.6$ & $<0.001$ * \\
\hline WHR & $0.84 \pm 0.07$ & $0.87 \pm 0.06$ & $0.80 \pm 0.07$ & $<0.001$ * \\
\hline Education level (\%) & & & & $<0.001$ * \\
\hline $\begin{array}{c}\text { Elementary school or } \\
\text { lower }\end{array}$ & 3.6 & 1.7 & 5.3 & \\
\hline Junior or senior school & 27.9 & 24.1 & 31.3 & \\
\hline College or higher & 68.5 & 74.1 & 63.4 & \\
\hline Income level (\%) & & & & $<0.001$ * \\
\hline$\leqq 20,000 \mathrm{NTD}$ & 21.0 & 15.0 & 26.4 & \\
\hline 20,001-40,000 NTD & 41.1 & 34.6 & 47.1 & \\
\hline$\geqq 40,001 \mathrm{NTD}$ & 38.0 & 50.4 & 26.5 & \\
\hline Marital status (\%) & & & & $<0.001$ * \\
\hline Never married & 54.2 & 52.9 & 55.4 & \\
\hline Married & 42.1 & 44.8 & 39.6 & \\
\hline Divorced/separated/widowed & 3.8 & 2.3 & 5.1 & \\
\hline $\begin{array}{c}\text { Self-reported health } \\
\text { status }(\%)\end{array}$ & & & & $<0.001$ * \\
\hline Excellent or good & 60.8 & 62.1 & 59.5 & \\
\hline Fair & 32.6 & 31.6 & 33.6 & \\
\hline Bad or poor & 6.6 & 6.3 & 6.9 & \\
\hline Smoking status (\%) & & & & $<0.001$ * \\
\hline Never & 83.8 & 70.5 & 95.7 & \\
\hline Current & 10.9 & 19.6 & 3.0 & \\
\hline Former & 5.4 & 9.9 & 1.2 & \\
\hline Chewing betel nut & & & & $<0.001$ * \\
\hline Never & 95.0 & 90.6 & 99.0 & \\
\hline Current & 2.1 & 3.5 & 0.8 & \\
\hline Former & 3.0 & 5.9 & 0.3 & \\
\hline
\end{tabular}

BMI, body mass index; NTD, New Taiwan dollar; SD, standard deviation; WC, waist circumference; WHR, waist-hip ratio. Values are expressed as means \pm SD. ${ }^{*} p<0.05$. 
Table 2. Body weight, height and BMI of men aged 23 to 64 years.

\begin{tabular}{|c|c|c|c|c|c|c|c|c|c|c|c|c|c|}
\hline Variables & $n$ & Mean & SD & $\mathrm{CV}$ & p5 & p10 & p15 & p25 & p50 & p75 & p85 & p90 & p95 \\
\hline \multicolumn{14}{|l|}{$\begin{array}{l}\text { Body weight } \\
\qquad(\mathrm{kg}) *+\end{array}$} \\
\hline $23-24$ & 2372 & $69.77^{\mathrm{a}, \mathrm{e}}$ & 11.46 & 0.16 & 53.0 & 56.0 & 58.0 & 61.3 & 69.0 & 77.0 & 82.0 & 86.0 & 91.0 \\
\hline $25-34$ & 8363 & $72.65^{b}$ & 10.87 & 0.15 & 56.0 & 59.0 & 61.0 & 65.0 & 72.0 & 80.0 & 84.0 & 87.0 & 93.0 \\
\hline $35-44$ & 8205 & $74.01^{c}$ & 10.30 & 0.14 & 58.0 & 61.0 & 63.0 & 67.0 & 74.0 & 81.0 & 85.0 & 88.0 & 92.0 \\
\hline $45-54$ & 6198 & $72.07^{\mathrm{d}}$ & 9.97 & 0.14 & 56.7 & 60.0 & 62.0 & 65.0 & 72.0 & 78.4 & 82.0 & 85.0 & 90.0 \\
\hline $55-64$ & 4547 & $69.55^{a, e}$ & 9.47 & 0.14 & 54.0 & 58.0 & 60.0 & 63.0 & 69.0 & 76.0 & 79.3 & 82.0 & 86.0 \\
\hline Total & 29,685 & 72.20 & 10.49 & 0.15 & 56.0 & 59.0 & 61.8 & 65.0 & 71.9 & 79.0 & 83.0 & 86.0 & 91.0 \\
\hline \multicolumn{14}{|l|}{$\underset{* \dagger}{\operatorname{Height}}(\mathrm{cm})$} \\
\hline $23-24$ & 2372 & $172.78^{a, b}$ & 5.86 & 0.03 & 164.0 & 166.0 & 167.0 & 169.0 & 173.0 & 177.0 & 179.0 & 180.0 & 182.0 \\
\hline $25-34$ & 8363 & $172.46^{a, b}$ & 5.93 & 0.03 & 163.0 & 165.0 & 166.1 & 169.0 & 172.0 & 176.0 & 178.0 & 180.0 & 182.0 \\
\hline $35-44$ & 8205 & $171.52^{c}$ & 6.01 & 0.04 & 162.0 & 164.0 & 165.1 & 168.0 & 172.0 & 175.9 & 178.0 & 179.0 & 181.0 \\
\hline $45-54$ & 6198 & $169.29^{d}$ & 6.08 & 0.04 & 159.0 & 162.0 & 163.0 & 166.0 & 169.0 & 173.0 & 175.0 & 177.0 & 179.0 \\
\hline $55-64$ & 4547 & $166.74^{\mathrm{e}}$ & 6.00 & 0.04 & 157.0 & 159.5 & 161.0 & 163.0 & 167.0 & 171.0 & 173.0 & 174.0 & 176.0 \\
\hline Total & 29,685 & 170.69 & 6.33 & 0.04 & 160.0 & 163.0 & 164.0 & 167.0 & 171.0 & 175.0 & 177.0 & 179.0 & 181.0 \\
\hline \multicolumn{14}{|c|}{$\underset{* \dagger}{\operatorname{BMI}}\left(\mathrm{kg} / \mathrm{m}^{2}\right)$} \\
\hline $23-24$ & 2372 & $23.35^{a}$ & 3.58 & 0.15 & 18.34 & 19.10 & 19.66 & 20.76 & 22.94 & 25.52 & 27.13 & 28.36 & 30.07 \\
\hline $25-34$ & 8363 & $24.41^{b}$ & 3.34 & 0.14 & 19.29 & 20.32 & 21.01 & 22.06 & 24.11 & 26.49 & 27.99 & 29.03 & 30.45 \\
\hline 35-44 & 8205 & $\begin{array}{c}25.14 \\
c, d, e\end{array}$ & 3.16 & 0.13 & 20.20 & 21.22 & 21.94 & 22.94 & 24.91 & 27.15 & 28.41 & 29.35 & 30.69 \\
\hline $45-54$ & 6198 & $\begin{array}{c}25.12 \\
c, d, e\end{array}$ & 3.08 & 0.12 & 20.38 & 21.38 & 22.04 & 23.04 & 24.98 & 27.01 & 28.30 & 29.06 & 30.48 \\
\hline $55-64$ & 4547 & $\begin{array}{c}24.99 \\
\mathrm{c}, \mathrm{d}, \mathrm{e}\end{array}$ & 2.97 & 0.12 & 20.43 & 21.45 & 22.07 & 23.05 & 24.84 & 26.83 & 28.04 & 28.84 & 30.11 \\
\hline Total & 29,685 & 24.77 & 3.25 & 0.13 & 19.71 & 20.76 & 21.47 & 22.53 & 24.58 & 26.81 & 28.08 & 29.05 & 30.45 \\
\hline
\end{tabular}

BMI, body mass index; CV, coefficient of variation; SD, standard deviation; $p$ with the ordinal number, percentiles. ${ }^{a, b, c, d, e}$ Superscripts on the mean values represent Tukey's test results. Means with the same letter represent that the mean values of the age groups have no significant difference between/among each other. In contrast, different superscript letters show significant differences $(p<0.05)$. * Significant differences in means were found between men and women (Student's $t$-test; $p<0.05$ ). ${ }^{+}$Significant differences in means were found across all age groups (ANOVA; $p<0.05$ ).

Table 3. WC, HC and WHR of men aged 23 to 64 years.

\begin{tabular}{cccccccccccccc}
\hline Variables & $n$ & Mean & SD & CV & p5 & p10 & p15 & p25 & p50 & p75 & p85 & p90 & p95 \\
\hline WC $(\mathrm{cm})^{* \dagger}$ & & & & & & & & & & & & \\
\hline $23-24$ & 2372 & $79.66^{\mathrm{a}}$ & 9.09 & 0.11 & 66.0 & 69.0 & 70.0 & 73.0 & 79.0 & 85.0 & 90.0 & 92.0 & 96.5 \\
\hline $25-34$ & 8363 & $82.84^{\mathrm{b}}$ & 8.67 & 0.10 & 70.0 & 72.0 & 74.0 & 77.0 & 82.0 & 89.0 & 92.0 & 94.0 & 98.0 \\
\hline $35-44$ & 8205 & $85.56^{\mathrm{c}, \mathrm{d}}$ & 8.37 & 0.10 & 72.0 & 75.0 & 77.0 & 80.0 & 85.0 & 91.0 & 94.0 & 97.0 & 100.0 \\
\hline $45-54$ & 6198 & $85.85^{\mathrm{c}, \mathrm{d}}$ & 8.18 & 0.10 & 73.0 & 75.0 & 77.5 & 80.0 & 86.0 & 91.0 & 94.0 & 96.0 & 100.0 \\
\hline $55-64$ & 4547 & $86.52^{\mathrm{e}}$ & 8.32 & 0.10 & 73.0 & 76.0 & 78.0 & 81.0 & 86.4 & 92.0 & 95.0 & 97.0 & 100.0 \\
\hline Total & 29,685 & 84.53 & 8.70 & 0.10 & 70.0 & 73.0 & 75.0 & 78.0 & 84.0 & 90.0 & 94.0 & 96.0 & 99.5 \\
\hline
\end{tabular}


Table 3. Cont.

\begin{tabular}{cccccccccccccc}
\hline Variables & $n$ & Mean & SD & CV & p5 & p10 & p15 & p25 & p50 & p75 & p85 & p90 & p95 \\
\hline HC $(\mathrm{cm})^{* \dagger}$ & & & & & & & & & & & & \\
\hline $23-24$ & 2372 & $95.92^{\mathrm{a}, \mathrm{e}}$ & 7.13 & 0.07 & 85.0 & 87.0 & 88.5 & 91.0 & 95.0 & 100.0 & 103.5 & 106.0 & 109.0 \\
\hline $25-34$ & 8363 & $97.37^{\mathrm{b}, \mathrm{c}}$ & 6.49 & 0.07 & 87.0 & 89.0 & 91.0 & 93.0 & 97.0 & 102.0 & 104.0 & 106.0 & 108.0 \\
\hline $35-44$ & 8205 & $97.60^{\mathrm{b}, \mathrm{c}}$ & 6.09 & 0.06 & 88.0 & 90.0 & 91.0 & 93.5 & 97.5 & 102.0 & 104.0 & 106.0 & 108.0 \\
\hline $45-54$ & 6198 & $96.47^{\mathrm{d}}$ & 5.87 & 0.06 & 87.0 & 89.0 & 90.5 & 92.5 & 96.0 & 100.0 & 102.0 & 104.0 & 106.5 \\
\hline $55-64$ & 4547 & $95.96^{\mathrm{a}, \mathrm{e}}$ & 5.78 & 0.06 & 87.0 & 89.0 & 90.0 & 92.0 & 96.0 & 100.0 & 102.0 & 103.0 & 106.0 \\
\hline Total & 29,685 & $96.92^{*}$ & 6.24 & 0.06 & 87.0 & 89.0 & 90.5 & 93.0 & 97.0 & 101.0 & 103.0 & 105.0 & 108.0 \\
\hline WHR * & & & & & & & & & & & & \\
\hline $23-24$ & 2372 & $0.83^{\mathrm{a}}$ & 0.05 & 0.06 & 0.75 & 0.76 & 0.78 & 0.79 & 0.82 & 0.86 & 0.89 & 0.90 & 0.93 \\
\hline $25-34$ & 8363 & $0.85^{\mathrm{b}}$ & 0.05 & 0.06 & 0.77 & 0.78 & 0.79 & 0.81 & 0.85 & 0.89 & 0.90 & 0.92 & 0.94 \\
\hline $35-44$ & 8205 & $0.88^{\mathrm{c}}$ & 0.05 & 0.06 & 0.79 & 0.81 & 0.82 & 0.84 & 0.88 & 0.91 & 0.93 & 0.94 & 0.96 \\
\hline $45-54$ & 6198 & $0.89^{\mathrm{d}}$ & 0.05 & 0.06 & 0.80 & 0.82 & 0.84 & 0.86 & 0.89 & 0.92 & 0.94 & 0.96 & 0.98 \\
\hline $55-64$ & 4547 & $0.90^{\mathrm{e}}$ & 0.06 & 0.07 & 0.81 & 0.83 & 0.85 & 0.87 & 0.90 & 0.94 & 0.96 & 0.97 & 0.99 \\
\hline Total & 29,685 & $0.87^{*}$ & 0.06 & 0.07 & 0.78 & 0.80 & 0.81 & 0.83 & 0.87 & 0.91 & 0.93 & 0.94 & 0.97 \\
\hline
\end{tabular}

BMI, body mass index; CV, coefficient of variation; SD, standard deviation; $\mathrm{p}$ with the ordinal number, percentiles. ${ }^{\mathrm{a}, \mathrm{b}, \mathrm{c}, \mathrm{d}, \mathrm{e}}$ Superscripts on the mean values represent Tukey's test results. Means with the same letter represent that the mean values of the age groups have no significant difference between/among each other. In contrast, different superscript letters show significant differences $(p<0.05)$. ${ }^{*}$ Significant differences in means were found between men and women (Student's $t$-test; $p<0.05$ ). ${ }^{\dagger}$ Significant differences in means were found across all age groups (ANOVA; $p<0.05$ ).

Table 4. Body weight, height and BMI of women aged 23 to 64 years.

\begin{tabular}{|c|c|c|c|c|c|c|c|c|c|c|c|c|c|}
\hline Variables & $n$ & Mean & SD & $\mathrm{CV}$ & p5 & p10 & p15 & p25 & p50 & p75 & p85 & p90 & p95 \\
\hline \multicolumn{14}{|l|}{$\begin{array}{l}\text { Body weight } \\
(\mathrm{kg})^{*+}\end{array}$} \\
\hline $23-24$ & 1993 & $55.53^{a}$ & 9.48 & 0.17 & 43.0 & 45.0 & 47.0 & 49.0 & 54.0 & 60.0 & 65.0 & 68.0 & 74.0 \\
\hline $25-34$ & 7554 & $56.46^{b}$ & 9.09 & 0.16 & 45.0 & 47.0 & 48.0 & 50.0 & 55.0 & 61.0 & 65.0 & 69.0 & 74.0 \\
\hline $35-44$ & 8915 & $\begin{array}{c}57.75 \\
\mathrm{c}, \mathrm{d}, \mathrm{e}\end{array}$ & 8.78 & 0.15 & 46.0 & 48.0 & 49.0 & 51.5 & 56.0 & 62.4 & 67.0 & 70.0 & 75.0 \\
\hline $45-54$ & 7551 & $\begin{array}{c}58.06 \\
\mathrm{c}, \mathrm{d}, \mathrm{e}\end{array}$ & 8.53 & 0.15 & 46.0 & 48.0 & 50.0 & 52.0 & 57.0 & 63.0 & 67.0 & 69.1 & 74.0 \\
\hline $55-64$ & 6888 & $\begin{array}{c}57.86 \\
\mathrm{c}, \mathrm{d}, \mathrm{e}\end{array}$ & 8.49 & 0.15 & 46.0 & 48.0 & 50.0 & 52.0 & 57.0 & 63.0 & 66.0 & 69.0 & 73.0 \\
\hline Total & 32,901 & 57.42 & 8.81 & 0.15 & 45.0 & 47.4 & 49.0 & 51.0 & 56.0 & 62.0 & 66.0 & 69.0 & 74.0 \\
\hline \multicolumn{14}{|l|}{$\underset{* \dagger}{\operatorname{Height}}(\mathrm{cm})$} \\
\hline $23-24$ & 1993 & $160.32^{a, b}$ & 5.81 & 0.04 & 151.0 & 153.0 & 154.6 & 156.0 & 160.0 & 164.0 & 166.0 & 168.0 & 170.0 \\
\hline $25-34$ & 7554 & $160.18^{a, b}$ & 5.68 & 0.04 & 151.0 & 153.0 & 154.0 & 156.0 & 160.0 & 164.0 & 166.0 & 167.5 & 170.0 \\
\hline $35-44$ & 8915 & $159.16^{c}$ & 5.46 & 0.03 & 150.0 & 152.0 & 154.0 & 155.5 & 159.0 & 163.0 & 165.0 & 166.0 & 168.0 \\
\hline $45-54$ & 7551 & $157.66^{d}$ & 5.40 & 0.03 & 149.0 & 151.0 & 152.0 & 154.0 & 158.0 & 161.0 & 163.0 & 164.8 & 166.9 \\
\hline $55-64$ & 6888 & $155.47^{\mathrm{e}}$ & 5.41 & 0.03 & 147.0 & 149.0 & 150.0 & 152.0 & 155.0 & 159.0 & 161.0 & 162.0 & 164.1 \\
\hline Total & 32,901 & 158.35 & 5.77 & 0.04 & 149.0 & 151.0 & 152.0 & 154.0 & 158.0 & 162.0 & 164.0 & 166.0 & 168.0 \\
\hline
\end{tabular}


Table 4. Cont.

\begin{tabular}{cccccccccccccc}
\hline Variables & $n$ & Mean & SD & CV & p5 & p10 & p15 & p25 & p50 & p75 & p85 & p90 & p95 \\
\hline $\begin{array}{c}\text { BMI }\left(\mathrm{kg} / \mathrm{m}^{2}\right) \\
*+\end{array}$ & & & & & & & & & & & & & \\
\hline $23-24$ & 1993 & $21.59^{\mathrm{a}}$ & 3.42 & 0.16 & 17.42 & 17.97 & 18.43 & 19.22 & 20.96 & 23.19 & 25.08 & 25.97 & 28.48 \\
\hline $25-34$ & 7554 & $22.01^{\mathrm{b}}$ & 3.39 & 0.15 & 17.72 & 18.43 & 18.90 & 19.71 & 21.36 & 23.53 & 25.24 & 26.64 & 28.76 \\
\hline $35-44$ & 8915 & $22.80^{\mathrm{c}}$ & 3.27 & 0.14 & 18.51 & 19.19 & 19.68 & 20.50 & 22.20 & 24.52 & 26.13 & 27.27 & 29.32 \\
\hline $45-54$ & 7551 & $23.36^{\mathrm{d}}$ & 3.25 & 0.14 & 18.83 & 19.65 & 20.17 & 21.05 & 22.94 & 25.15 & 26.62 & 27.70 & 29.37 \\
\hline $55-64$ & 6888 & $23.94^{\mathrm{e}}$ & 3.29 & 0.14 & 19.20 & 20.16 & 20.70 & 21.64 & 23.55 & 25.89 & 27.21 & 28.25 & 29.90 \\
\hline Total & 32,901 & 22.91 & 3.39 & 0.15 & 18.29 & 19.05 & 19.63 & 20.54 & 22.38 & 24.80 & 26.37 & 27.48 & 29.36 \\
\hline
\end{tabular}

BMI, body mass index; CV, coefficient of variation; SD, standard deviation; $p$ with the ordinal number, percentiles. a,b,c,d,e Superscripts on the mean values represent Tukey's test results. Means with the same letter represent that the mean values of the age groups have no significant difference between/among each other. In contrast, different superscript letters show significant differences $(p<0.05)$. * Significant differences in means were found between men and women (Student's $t$-test; $p<0.05) .{ }^{+}$Significant differences in means were found across all age groups (ANOVA; $p<0.05)$.

Table 5. WC, HC and WHR of women aged 23 to 64 years.

\begin{tabular}{|c|c|c|c|c|c|c|c|c|c|c|c|c|c|}
\hline Variables & $n$ & Mean & SD & $\mathrm{CV}$ & p5 & p10 & p15 & p25 & p50 & p75 & p85 & p90 & p95 \\
\hline \multicolumn{14}{|l|}{$\mathrm{WC}(\mathrm{cm}) * \dagger$} \\
\hline $23-24$ & 1993 & $71.80^{a}$ & 8.45 & 0.12 & 61.0 & 62.0 & 64.0 & 66.0 & 70.0 & 76.5 & 80.0 & 83.5 & 88.0 \\
\hline $25-34$ & 7554 & $73.23^{b}$ & 8.57 & 0.12 & 62.0 & 63.5 & 65.0 & 67.0 & 72.0 & 78.0 & 82.0 & 85.0 & 89.5 \\
\hline $35-44$ & 8915 & $75.27^{c}$ & 8.49 & 0.11 & 63.5 & 65.5 & 67.0 & 69.0 & 74.0 & 80.0 & 84.0 & 87.0 & 91.0 \\
\hline $45-54$ & 7551 & $77.01^{d}$ & 8.47 & 0.11 & 64.5 & 67.0 & 68.5 & 71.0 & 76.0 & 82.0 & 86.0 & 88.0 & 92.0 \\
\hline $55-64$ & 6888 & $79.41^{\mathrm{e}}$ & 8.75 & 0.11 & 66.0 & 69.0 & 70.5 & 73.0 & 79.0 & 85.0 & 88.5 & 91.0 & 95.0 \\
\hline Total & 32,901 & 75.86 & 8.88 & 0.12 & 63.0 & 65.0 & 67.0 & 69.5 & 75.0 & 81.0 & 85.0 & 88.0 & 92.0 \\
\hline \multicolumn{14}{|l|}{$\mathrm{HC}(\mathrm{cm})^{* \dagger}$} \\
\hline $23-24$ & 1993 & $92.89^{a}$ & 7.01 & 0.08 & 82.5 & 85.0 & 86.0 & 88.0 & 92.0 & 97.0 & 100.0 & 102.0 & 106.0 \\
\hline $25-34$ & 7554 & $93.66^{b}$ & 6.87 & 0.07 & 84.0 & 86.0 & 87.0 & 89.0 & 93.0 & 98.0 & 100.5 & 103.0 & 106.0 \\
\hline $35-44$ & 8915 & $94.29^{c, d}$ & 6.50 & 0.07 & 85.0 & 87.0 & 88.0 & 90.0 & 94.0 & 98.0 & 101.0 & 103.0 & 106.0 \\
\hline $45-54$ & 7551 & $94.40^{c, d}$ & 6.40 & 0.07 & 85.0 & 87.0 & 88.0 & 90.0 & 94.0 & 98.0 & 101.0 & 103.0 & 106.0 \\
\hline $55-64$ & 6888 & $94.71^{\mathrm{e}}$ & 6.42 & 0.07 & 85.0 & 87.0 & 88.0 & 90.0 & 94.0 & 99.0 & 101.0 & 103.0 & 106.0 \\
\hline Total & 32,901 & 94.17 & 6.59 & 0.07 & 84.0 & 86.0 & 88.0 & 90.0 & 94.0 & 98.0 & 101.0 & 103.0 & 106.0 \\
\hline \multicolumn{14}{|l|}{ WHR *t } \\
\hline $23-24$ & 1993 & $0.77^{\mathrm{a}}$ & 0.06 & 0.08 & 0.69 & 0.70 & 0.71 & 0.73 & 0.76 & 0.81 & 0.84 & 0.85 & 0.89 \\
\hline $25-34$ & 7554 & $0.78^{b}$ & 0.06 & 0.08 & 0.69 & 0.71 & 0.72 & 0.74 & 0.77 & 0.82 & 0.85 & 0.86 & 0.89 \\
\hline $35-44$ & 8915 & $0.80^{\mathrm{c}}$ & 0.06 & 0.08 & 0.71 & 0.73 & 0.74 & 0.76 & 0.79 & 0.84 & 0.86 & 0.88 & 0.90 \\
\hline $45-54$ & 7551 & $0.82^{d}$ & 0.06 & 0.07 & 0.72 & 0.74 & 0.75 & 0.77 & 0.81 & 0.85 & 0.88 & 0.89 & 0.92 \\
\hline $55-64$ & 6888 & $0.84^{\mathrm{e}}$ & 0.06 & 0.07 & 0.74 & 0.76 & 0.77 & 0.80 & 0.83 & 0.88 & 0.90 & 0.92 & 0.95 \\
\hline Total & 32,901 & 0.80 & 0.07 & 0.09 & 0.71 & 0.72 & 0.74 & 0.76 & 0.80 & 0.85 & 0.87 & 0.89 & 0.92 \\
\hline
\end{tabular}

BMI, body mass index; CV, coefficient of variation; SD, standard deviation; $\mathrm{p}$ with the ordinal number, percentiles. ${ }^{\mathrm{a}, \mathrm{b}, \mathrm{c}, \mathrm{d}, \mathrm{e}}$ Superscripts on the mean values represent Tukey's test results. Means with the same letter represent that the mean values of the age groups have no significant difference between/among each other. In contrast, different superscript letters show significant differences $(p<0.05)$. ${ }^{*}$ Significant differences in means were found between men and women (Student's $t$-test; $p<0.05$ ). ${ }^{\dagger}$ Significant differences in means were found across all age groups (ANOVA; $p<0.05)$.

The difference in the mean body weight between the youngest (23-24 years) and oldest (55-64 years) age groups was $0.22 \mathrm{~kg}$ (median $0 \mathrm{~kg}$ ) in men and $2.33 \mathrm{~kg}$ (median $3 \mathrm{~kg}$ ) 
in women. Tukey's multiple comparisons test revealed no significant difference between the youngest and oldest age groups. However, the 23-24-year age group was significantly different from all other age groups in men, and the 23-24-year and 25-34-year age groups were significantly different from all the other age groups in women.

The difference in the mean height between the youngest (23-24 years) and oldest (55-64 years) age groups was $6.04 \mathrm{~cm}$ (median $6 \mathrm{~cm}$ ) in men and $4.85 \mathrm{~cm}$ (median $5 \mathrm{~cm}$ ) in women. Tukey's multiple comparisons test revealed a significant difference between the three older age groups (35-44, 45-54 and 55-64 years) and the two younger age groups (23-24 and 25-34 years) in both men and women. Moreover, the CVs for height were around 0.03 to 0.04 in both men and women. These results presented even distributions in height in the study population (Tables 2 and 4 ).

In men, the difference in the mean BMI was $1.64 \mathrm{~kg} / \mathrm{m}^{2}$ (median $1.9 \mathrm{~kg} / \mathrm{m}^{2}$ ) between the youngest (23-24 years) and oldest (55-64 years) age groups. Tukey's multiple comparisons test revealed a significant difference between the two younger age groups (23-24 and 25-34 years) and the three older age groups (35-44, 45-54 and 55-64 years). In women, the difference in the mean BMI was $2.35 \mathrm{~kg} / \mathrm{m}^{2}$ (median $2.59 \mathrm{~kg} / \mathrm{m}^{2}$ ) between the youngest and the oldest age groups. There were statistically significant differences in all age groups.

The difference in the mean WC between the youngest (23-24 years) and oldest (55-64 years) age groups was $6.86 \mathrm{~cm}$ (median $7.4 \mathrm{~cm}$ ) in men and $7.61 \mathrm{~cm}$ (median $9 \mathrm{~cm}$ ) in women. In both men and women, the WC increased with age. Tukey's multiple comparisons test showed a significant difference between the two younger age groups (35-44 and $45-54$ years) and the three older age groups (35-44, 45-54 and 55-64 years). However, there was no significant difference between the 35-44-year and the 45-54-year age group in men (Table 3), while there were significant differences between all the age groups in women (Table 5).

The difference in the mean HC between the youngest (23-24 years) and oldest (55-64 years) age groups was $0.04 \mathrm{~cm}$ (median $1 \mathrm{~cm}$ ) in men and $1.82 \mathrm{~cm}$ (median $2 \mathrm{~cm}$ ) in women. The HC increased with age in women. Tukey's multiple comparisons test showed that the 45-54-year age group was significantly different from all other age groups in men, while there were significant differences between all age groups in women (Tables 4 and 5).

The difference in the mean WHR between the youngest (23-24 years) and oldest (55-64 years) age groups was $0.07 \mathrm{~cm}$ (median $0.08 \mathrm{~cm}$ ) in men and $0.07 \mathrm{~cm}$ (median $0.07 \mathrm{~cm}$ ) in women. In both men and women, the WHR increased with age. Moreover, there was a significant difference between all age groups in both men and women (Tables 4 and 5).

Table 6 presents the prevalence of BMI categories according to the Taiwanese cutoff points, showing that in both men and women, the normal BMI category was most prevalent (men $40.69 \%$, women $61.72 \%$ ). However, the percentages of overweight and obese individuals combined were larger in men (57.56\%) than in women $(32.17 \%)$. Looking at BMI categories by age group, it is clear that in both men and women, the highest percentages of underweight people (men $56.28 \%$, women $15.81 \%$ ) were found in the youngest age group (23-24 years) and the highest percentages of overweight people (men $38.69 \%$, women $28.76 \%$ ) were found in the oldest age group (55-64 years). In addition, the highest percentages of obese people were found in the oldest age group in women $(16.45 \%)$ and in the $45-54$-year age group in men (25.04\%). 
Table 6. BMI in different categories of men and women aged 23 to 64 years.

\begin{tabular}{|c|c|c|c|c|c|c|c|c|c|c|c|c|}
\hline \multirow{3}{*}{$\begin{array}{c}\text { Variables } \\
\text { Men * }\end{array}$} & \multicolumn{12}{|c|}{ Age Groups (Years) } \\
\hline & \multicolumn{2}{|c|}{$23-24$} & \multicolumn{2}{|c|}{$25-34$} & \multicolumn{2}{|c|}{$35-44$} & \multicolumn{2}{|c|}{$45-54$} & \multicolumn{2}{|c|}{$55-64$} & \multicolumn{2}{|c|}{ Total } \\
\hline & $n$ & $\%$ & $n$ & $\%$ & $n$ & $\%$ & $n$ & $\%$ & $n$ & $\%$ & $n$ & $\%$ \\
\hline $\begin{array}{l}\text { Underweight } \\
<18.5\left(\mathrm{~kg} / \mathrm{m}^{2}\right)\end{array}$ & 136 & 5.73 & 184 & 2.20 & 82 & 1.00 & 66 & 1.06 & 51 & 1.12 & 519 & 1.75 \\
\hline $\begin{array}{c}\text { Normal } \\
18.5-23.9 \\
\left(\mathrm{~kg} / \mathrm{m}^{2}\right)\end{array}$ & 1335 & 56.28 & 3886 & 46.47 & 2977 & 36.28 & 2194 & 35.40 & 1686 & 37.08 & 12,078 & 40.69 \\
\hline $\begin{array}{c}\text { Overweight } \\
24.0-26.9 \\
\left(\mathrm{~kg} / \mathrm{m}^{2}\right)\end{array}$ & 523 & 22.05 & 2510 & 30.01 & 2963 & 36.11 & 2386 & 38.50 & 1759 & 38.69 & 10,141 & 34.16 \\
\hline $\begin{array}{c}\text { Obese } \\
\geq 27\left(\mathrm{~kg} / \mathrm{m}^{2}\right)\end{array}$ & 378 & 15.94 & 1783 & 21.32 & 2183 & 26.61 & 1552 & 25.04 & 1051 & 23.11 & 6947 & 23.40 \\
\hline Total & 2372 & & 8363 & & 8205 & & 6198 & & 4547 & & 29,685 & \\
\hline \multicolumn{13}{|l|}{ Women * } \\
\hline $\begin{array}{l}\text { Underweight } \\
<18.5\left(\mathrm{~kg} / \mathrm{m}^{2}\right)\end{array}$ & 315 & 15.81 & 812 & 10.75 & 443 & 4.97 & 258 & 3.42 & 183 & 2.66 & 2011 & 6.11 \\
\hline $\begin{array}{c}\text { Normal } \\
18.5-23.9 \\
\left(\mathrm{~kg} / \mathrm{m}^{2}\right)\end{array}$ & 1297 & 65.07 & 5079 & 67.23 & 5827 & 65.36 & 4512 & 59.75 & 3591 & 52.13 & 20,306 & 61.72 \\
\hline $\begin{array}{c}\text { Overweight } \\
24.0-26.9 \\
\left(\mathrm{~kg} / \mathrm{m}^{2}\right)\end{array}$ & 224 & 11.24 & 996 & 13.19 & 1650 & 18.51 & 1815 & 24.04 & 1981 & 28.76 & 6666 & 20.26 \\
\hline $\begin{array}{c}\text { Obese } \\
\geq 27\left(\mathrm{~kg} / \mathrm{m}^{2}\right)\end{array}$ & 157 & 7.88 & 667 & 8.83 & 995 & 11.16 & 966 & 12.79 & 1133 & 16.45 & 3918 & 11.91 \\
\hline Total & 1993 & & 7554 & & 8915 & & 7551 & & 6888 & & 32,901 & \\
\hline \multicolumn{13}{|l|}{ Pooled * } \\
\hline $\begin{array}{l}\text { Underweight } \\
<18.5\left(\mathrm{~kg} / \mathrm{m}^{2}\right)\end{array}$ & 451 & 10.33 & 996 & 6.26 & 525 & 3.07 & 324 & 2.36 & 234 & 2.05 & 2530 & 4.04 \\
\hline $\begin{array}{c}\text { Normal } \\
18.5-23.9 \\
\left(\mathrm{~kg} / \mathrm{m}^{2}\right)\end{array}$ & 2632 & 60.30 & 8965 & 56.32 & 8804 & 51.42 & 6706 & 48.78 & 5277 & 46.14 & 32,384 & 51.75 \\
\hline $\begin{array}{c}\text { Overweight } \\
24.0-26.9 \\
\left(\mathrm{~kg} / \mathrm{m}^{2}\right)\end{array}$ & 747 & 17.11 & 3506 & 22.03 & 4613 & 26.95 & 4201 & 30.55 & 3740 & 32.71 & 16,807 & 26.85 \\
\hline $\begin{array}{c}\text { Obese } \\
\geq 27\left(\mathrm{~kg} / \mathrm{m}^{2}\right)\end{array}$ & 535 & 12.26 & 2450 & 15.39 & 3178 & 18.56 & 2518 & 18.31 & 2184 & 19.10 & 10,865 & 17.36 \\
\hline Total & 4365 & & 15,917 & & 17,120 & & 13,749 & & 11,435 & & 62,586 & \\
\hline
\end{tabular}

BMI, body mass index. ${ }^{*}$ Significant differences in means were found across all age groups $\left(x^{2}\right.$ test; $\left.p<0.05\right)$.

Table 7 shows that men had a higher proportion of a normal WC (71.93\%) than women $(69.35 \%)$ but a lower normal WHR $(69.44 \%)$ than women $(76.90 \%)$. Both men and women in the youngest age group (23-24 years) had the highest percentages of a normal WC (men $84.95 \%$, women $83.74 \%$ ) and WHR (men $89.71 \%$, women $89.01 \%$ ). The oldest age group (55-64 years) had higher percentages of obese people (WC: men 35.01\%, women 46.56\%; WHR: men 50.56\%, women 39.85\%). 
Table 7. WC and WHR in different categories of men and women aged 23 to 64 years.

\begin{tabular}{|c|c|c|c|c|c|c|c|c|c|c|c|c|}
\hline \multicolumn{13}{|c|}{ Age Groups } \\
\hline Variables & \multicolumn{2}{|c|}{$23-24$} & \multicolumn{2}{|c|}{$25-34$} & \multicolumn{2}{|c|}{$35-44$} & \multicolumn{2}{|c|}{$45-54$} & \multicolumn{2}{|c|}{$55-64$} & \multicolumn{2}{|c|}{ Total } \\
\hline Men * & $n$ & $\%$ & $n$ & $\%$ & $n$ & $\%$ & $n$ & $\%$ & $n$ & $\%$ & $N$ & $\%$ \\
\hline $\mathrm{WC}<90(\mathrm{~cm})$ & 2015 & 84.95 & 6484 & 77.53 & 5654 & 68.91 & 4243 & 68.46 & 2955 & 64.99 & 21,351 & 71.93 \\
\hline $\mathrm{WC} \geqq 90(\mathrm{~cm})$ & 357 & 15.05 & 1879 & 22.47 & 2551 & 31.09 & 1955 & 31.54 & 1592 & 35.01 & 8334 & 28.07 \\
\hline Total & 2372 & & 8363 & & 8205 & & 6198 & & 4547 & & 29,685 & \\
\hline \multicolumn{13}{|l|}{ Women * } \\
\hline $\mathrm{WC}<80(\mathrm{~cm})$ & 1669 & 83.74 & 5979 & 79.15 & 6512 & 73.05 & 4976 & 65.90 & 3681 & 53.44 & 22,817 & 69.35 \\
\hline $\mathrm{WC} \geqq 80(\mathrm{~cm})$ & 324 & 16.26 & 1575 & 20.85 & 2403 & 26.95 & 2575 & 34.10 & 3207 & 46.56 & 10,084 & 30.65 \\
\hline Total & 1993 & & 7554 & & 8915 & & 7551 & & 6888 & & 32,901 & \\
\hline \multicolumn{13}{|l|}{ Pooled * } \\
\hline $\begin{array}{l}\text { WC below } \\
\text { cut-off }\end{array}$ & 3684 & 84.40 & 12,463 & 78.30 & 12,166 & 71.06 & 9219 & 67.05 & 6636 & 58.03 & 44,168 & 70.57 \\
\hline WC above cut-off & 681 & 15.60 & 3454 & 21.70 & 4954 & 28.94 & 4530 & 32.95 & 4799 & 41.97 & 18,418 & 29.43 \\
\hline Total & 4365 & & 15,917 & & 17,120 & & 13,749 & & 11,435 & & 62,586 & \\
\hline \multicolumn{13}{|l|}{ Men * } \\
\hline $\mathrm{WHR} \leqq 0.90$ & 2128 & 89.71 & 6942 & 83.01 & 5656 & 68.93 & 3639 & 58.71 & 2248 & 49.44 & 20,613 & 69.44 \\
\hline WHR $>0.90$ & 244 & 10.29 & 1421 & 16.99 & 2549 & 31.07 & 2559 & 41.29 & 2299 & 50.56 & 9072 & 30.56 \\
\hline Total & 2372 & & 8363 & & 8205 & & 6198 & & 4547 & & 29,685 & \\
\hline \multicolumn{13}{|l|}{ Women * } \\
\hline $\mathrm{WHR} \leqq 0.85$ & 1774 & 89.01 & 6525 & 86.38 & 7231 & 81.11 & 5629 & 74.55 & 4143 & 60.15 & 25,302 & 76.90 \\
\hline WHR > 0.85 & 219 & 10.99 & 1029 & 13.62 & 1684 & 18.89 & 1922 & 25.45 & 2745 & 39.85 & 7599 & 23.10 \\
\hline Total & 1993 & & 7554 & & 8915 & & 7551 & & 6888 & & 32,901 & \\
\hline \multicolumn{13}{|l|}{ Pooled * } \\
\hline $\begin{array}{l}\text { WHR below } \\
\text { cut-off }\end{array}$ & 3902 & 89.39 & 13,467 & 84.61 & 12,887 & 75.27 & 9268 & 67.41 & 6391 & 55.89 & 45,915 & 73.36 \\
\hline $\begin{array}{c}\text { WHR above } \\
\text { cut-off }\end{array}$ & 463 & 10.61 & 2450 & 15.39 & 4233 & 24.73 & 4481 & 32.59 & 5044 & 44.11 & 16,671 & 26.64 \\
\hline Total & 4365 & & 15,917 & & 17,120 & & 13,749 & & 11,435 & & 62,586 & \\
\hline
\end{tabular}

WC, waist circumference; WHR, waist-hip ratio. ${ }^{*}$ Significant differences in means were found across all age groups $\left(x^{2}\right.$ test; $\left.p<0.05\right)$.

\section{Discussion}

The purpose of the present study was to provide reference data of gender- and agespecific distributions in Taiwanese adults' anthropometric parameters. Results showed significant differences in most anthropometric outcomes (weight, height, BMI, WC, HC and WHR) between genders. More importantly, at the weight level, the prevalence of underweight people was $1.75 \%$ for men and $6.11 \%$ for women, and the prevalence decreased with age. Specifically, there were significant differences in the height, BMI and WC between the youngest and oldest age groups $(p<0.05)$ in women. Differences in the WHR were significant between all age groups.

In anthropometric outcomes, results indicated that all indexes (weight, height, BMI, WC, HC and WHR) of men and women were significantly different in each age group. In addition, men are higher means than women, consistent with previous findings. In a previous study [31], all body dimensions were manually measured using digital calipers and measuring tapes in 100 adults and 100 older people, and the results were the same as the present study. In addition, the mean values in both men and women were significantly different in every age group. In men, the mean WC and WHR were higher in the oldest than 
the youngest age group. In women, except those for body weight, all the variable means were higher in the oldest age group. This is because aging affects the body composition and metabolism differently between genders, leading to reduced fat oxidation and accumulation of upper-body fat in men and an increased ratio of upper-lower body fat and bone loss in women [32].

WHR results indicated that obesity rates are higher in older people than in the younger population in both men and women. According to the WHO [30], individuals with a higher WHR may have higher abdominal obesity risk. Abdominal obesity is significantly associated with cardiovascular disease [33], risk of cancer [34,35], all-cause mortality [36] and metabolic syndrome [37]. Practitioners should understand that a high WHR could reveal possible health risks for their clients. In addition, promoting a healthier lifestyle could be essential for this population.

On the other hand, although the CVs showed minor dispersions among groups, and the examiners were trained and qualified in the courses, the absolute reliability of the measurements could not be further tested based on the current cross-sectional data. Future studies should be aware of the test reliabilities. For instance, a repeated test may be conducted for the calculation of individual CVs. Thus, the mean CV can be applied to compare the reliability among the measurements [38]. A small mean CV represents a better consistency within each measurement [39].

The strength of the present study was a representative sample from Taiwanese adults. However, there were some limitations. First, the study adopted a cross-sectional design. Thus, no causal relationship could be guaranteed. Future studies should focus on a longitudinal study design to examine sex- and age-related effects on anthropometric development. Second, this study recruited 23-64-year-old Taiwanese adults and cannot be effectively estimated to other populations, such as different ages, races and cultures. Therefore, future studies should survey data from different population groups [40,41] to build a more comprehensive anthropometric profile. Third, as mentioned earlier, although data Heteroscedasticity seemed acceptable by the $\mathrm{CV}$, a measurement error may exist. Lastly, the NPFSIT should widely collect the background of its participants, include more scientific surveys and allow users to connect the data with other sources (e.g., health insurance, medical history) in order to create a better, more comprehensive platform for researchers.

\section{Conclusions}

The anthropometric status provides a preliminary evaluation of one's health and warrants a suitable profile for reference. The present study used a representative population of Taiwanese adults for analysis and provided details of the anthropometric distribution. Even though differences among different ages and genders have been previously reported, the results provide sufficient profiles to practitioners in Taiwan for both clinical and theoretical purposes.

Author Contributions: S.-C.C. drafted the original manuscript. C.-W.L. and P.-F.L. participated in the design and conducted and supervised statistical analyses. H.-L.C. and C.-C.H. critically reviewed and modified the manuscript. All authors have read and agreed to the published version of the manuscript.

Funding: The research was funded by the Taiwan Ministry of Science and Technology (MOST 107-2627-M-030-002) and the Taiwan Ministry of Education (FJU-A0108153).

Institutional Review Board Statement: The study design and analysis protocol were supervised by the Institutional Review Board of the Fu Jen Catholic University, Taiwan (FJU-IRB C108006).

Informed Consent Statement: The informed consent has been provided before data collection.

Data Availability Statement: The data used in this study is domestically available for public researches. Users may use the data at the appointed institute after the approval from the Sports Cloud: 
Information and Application Research Center of Sports for All, Sport Administration, Ministry of Education, Taiwan.

Acknowledgments: The data for this study were provided by the Sports Cloud: Information and Application Research Center of Sports for All, Sport Administration, Ministry of Education, Taiwan. The authors and their interpretations do not represent those of the Sport Administration, Taiwan.

Conflicts of Interest: The authors declare no conflict of interest.

\section{References}

1. National Development Council. Population Estimates of the Republic of China (2020 to 2070). Available online: https://www. ndc.gov.tw/Content_List.aspx?n=3CF120A42CD31054 (accessed on 27 August 2020).

2. Heller, P.S. The challenge of an aged and shrinking population: Lessons to be drawn from Japan's experience. J. Econ. Ageing 2016, 8, 85-93. [CrossRef]

3. Hsieh, K.Y.C.; Tung, A.C. Taiwan's National Pension Program: A remedy for rapid population aging? J. Econ. Ageing 2016, 8, 52-66. [CrossRef]

4. Lee, S.H.; Ogawa, N.; Matsukura, R. Japan's pension reform, labor market responses, and savings. J. Econ. Ageing 2016, 8, 67-75. [CrossRef]

5. Mitchell, O.S.; Mukherjee, A. Assessing the demand for micro pensions among India's poor. J. Econ. Ageing 2017, 9, 30-40. [CrossRef]

6. Cristea, M.; Noja, G.G.; Stefea, P.; Sala, A.L. The impact of population aging and public health support on EU labor markets. Int. J. Environ. Res. Public Health 2020, 17, 1439. [CrossRef] [PubMed]

7. Towlson, C.; Cobley, S.; Parkin, G.; Lovell, R. When does the influence of maturation on anthropometric and physical fitness characteristics increase and subside? Scand. J. Med. Sci. Sports 2018, 28, 1946-1955. [CrossRef]

8. Munro, H.N. Nutrition and aging. Br. Med. Bull. 1981, 37, 83-88. [CrossRef] [PubMed]

9. Arnaoutis, G.; Georgoulis, M.; Psarra, G.; Milkonidou, A.; Panagiotakos, D.B.; Kyriakou, D.; Bellou, E.; Tambalis, K.D.; Sidossis, L.S. Association of anthropometric and lifestyle parameters with fitness levels in Greek schoolchildren: Results from the EYZHN Program. Front. Nutr. Obes. 2018, 5, 10. [CrossRef]

10. Newman, A.B.; Yanez, D.; Harris, T.; Duzbury, A.; Enright, P.L.; Fried, L.P. Cardiovascular Study Research Group: Weight change in old age and its association with mortality. J. Am. Geriatr. Soc. 2001, 49, 1309-1318. [CrossRef]

11. Miller, M.D.; Crotty, M.; Giles, L.C.; Bannerman, E.; Whitehead, C.; Cobiac, L.; Daniels, L.A.; Andrews, G. Corrected arm muscle area: An independent predictor of long-term mortality in community-dwelling older adults? J. Am. Geriatr. Soc. 2002, 50, 1272-1277. [CrossRef] [PubMed]

12. Perissinotto, E.; Pisent, C.; Sergi, G.; Grigoletto, F.; Enzi, G.; ILSA Working Group. Anthropometric measurements in the elderly: Age and gender differences. Br. J. Nutr. 2002, 87, 177-186. [CrossRef]

13. Hootman, K.C.; Guertin, K.A.; Cassano, P.A. Stress and psychological constructs related to eating behavior are associated with anthropometry and body composition in young adults. Appetite 2018, 125, 287-294. [CrossRef] [PubMed]

14. Wang, E.M.Y.; Wang, M.J.; Yeh, W.Y.; Shih, Y.C.; Lin, Y.C. Development of anthropometric work environment for Taiwanese workers. Int. J. Ind. Ergon. 1999, 23, 3-8. [CrossRef]

15. Vyavahare, R.T.; Kallurkar, S.P. Anthropometry of male agricultural workers of western India for the design of tools and equipments. Int. J. Ind. Ergon. 2016, 53, 80-85. [CrossRef]

16. Tavana, M.; Kazemi, M.R.; Vafadarnikjoo, A.; Mobin, M. An artificial immune algorithm for ergonomic product classification using anthropometric measurements. Measurement 2016, 94, 621-629. [CrossRef]

17. Nicholas, D.; Paul, H.; Peter, W. Establishing metrics for the evaluation of touch screen kiosks. J. Inf. Sci. 2001, $27,61-71$. [CrossRef]

18. De Onis, M.; Habicht, J.P. Anthropometric reference data for international use: Recommendations from a World Health Organization Expert Committee. Am. J. Clin. Nutr. 1996, 64, 650-658. [CrossRef]

19. World Health Organization. Physical Status: The Use and Interpretation of Anthropometry; Report of a WHO Expert Committee; Technical Report Series; World Health Organization: Geneva, Switzerland, 1995; Volume 854.

20. World Health Organization. Use and interpretation of anthropometric indicators of nutritional status. Bull. World Health Organ. 1986, 64, 929-941.

21. Lin, J.D.; Yen, C.F.; Wu, J.L. Patterns of obesity among children and adolescents with intellectual disabilities in Taiwan. J. Appl. Res. Intellect. Disabil. 2005, 18, 123-129. [CrossRef]

22. Hou, M.T.; Wu, P.Y.; Lin, Y.B.; Chang, Y.F.; Chen, C.Y.; Guan, D.S.; Yang, Y.C.; Wu, C.H. Physical Fitness Performance and Normative Score of Elderly Male Rural Dwellers. Taiwan Geriatr. Gerontol. 2011, 6, 48-60.

23. Lee, P.F.; Ho, C.C.; Yeh, D.P.; Hung, C.T.; Chang, Y.C.; Liu, C.C.; Tseng, C.Y.; Hsieh, X.Y. Cross-sectional associations of physical fitness performance level and sleep duration among older adults: Results from the national physical fitness survey in Taiwan. Int. J. Environ. Res. Public Health 2020, 17, 388. [CrossRef] [PubMed]

24. Lin, Y.T.; Lee, P.F.; Lee, T.S.; Ho, C.C. Poor physical fitness performance as a predictor of general adiposity in Taiwanese adults. Int. J. Environ. Res. Public Health 2020, 17, 2686. [CrossRef] 
25. Chen, H.L.; Lee, P.F.; Chang, Y.C.; Hsu, F.S.; Tseng, C.Y.; Hsieh, X.Y.; Ho, C.C. The association between physical fitness performance and subjective happiness among Taiwanese adults. Int. J. Environ. Res. Public Health 2020, 17, 3774. [CrossRef]

26. Liao, Y.; Tsai, H.H.; Wang, H.S.; Ling, C.P.; Wu, M.C.; Chen, J.F. Traveling by private motorized vehicle and physical fitness in Taiwanese adults. Int. J. Behav. Med. 2016, 23, 395-401. [CrossRef]

27. Liu, C.M.; Lin, K.F. Estimation of VO2max: A comparative analysis of post-exercise heart rate and physical fitness index from 3-minute step test. JESF 2007, 5, 118-123.

28. Li, W.C.; Chen, I.C.; Chang, Y.C.; Loke, S.S.; Wang, S.H.; Hsiao, K.Y. Waist-to-height ratio, waist circumference, and body mass index as indices of cardiometabolic risk among 36,542 Taiwanese adults. Eur. J. Nutr. 2013, 52, 57-65. [CrossRef] [PubMed]

29. Health Promotion Administration, Ministry of Health and Welfare. Check Your Body Weight Every Day. Available online: https:/ / www.hpa.gov.tw/Home/Index.aspx (accessed on 15 August 2020).

30. World Health Organization. Waist Circumference and Waist-Hip Ratio. In Proceedings of the WHO Expert Consultation, Geneva, Switzerland, 8-11 December 2008.

31. Lee, Y.C.; Chen, C.H.; Lee, C.H. Body anthropometric measurements of Singaporean adult and elderly population. Measurement 2019, 148, 106949. [CrossRef]

32. Horber, F.F.; Gruber, B.; Thomi, F.; Jensen, E.X.; Jaeger, P. Effect of sex and age on bone mass, body composition and fuel metabolism in humans. Nutrition 1997, 13, 524-534. [CrossRef]

33. Fan, H.; Li, X.; Zheng, L.; Chen, X.; Ian, Q.; Wu, H.; Ding, X.; Qian, D.; Shen, Y.; Yu, Z.; et al. Abdominal obesity is strongly associated with cardiovascular disease and its risk factors in elderly and very elderly community-dwelling Chinese. Sci. Rep. 2016, 6, 21521. [CrossRef]

34. Dong, Y.; Zhou, J.; Zhu, Y.; Luo, L.; He, T.; Hu, H.; Liu, H.; Zhang, Y.; Luo, D.; Xu, S.; et al. Abdominal obesity and colorectal cancer risk: Systematic review and meta-analysis of prospective studies. Biosci. Rep. 2017, 37, BSR20170945. [CrossRef]

35. Hidayat, K.; Du, X.; Chen, G.; Shi, M.; Shi, B. Abdominal obesity and lung cancer risk: Systematic review and meta-analysis of prospective studies. Nutrients 2016, 8, 810. [CrossRef] [PubMed]

36. David, C.N.; Mello, R.B.; Bruscato, N.M.; Moriguchi, E.H. Overweight and abdominal obesity association with all-cause and cardiovascular mortality in the elderly aged 80 and over: A cohort study. J. Nutr. Health Aging 2017, 21, 597-603. [CrossRef]

37. Després, J.P.; Lemieux, I. Abdominal obesity and metabolic syndrome. Nature 2006, 444, 881-887. [CrossRef]

38. Atkinson, G.; Nevill, A.M. Statistical methods for assessing measurement error (reliability) in variables relevant to sports medicine. Sports Med. 1998, 26, 217-238. [CrossRef] [PubMed]

39. Shechtman, O. The Coefficient of Variation as an Index of Measurement Reliability. In Methods of Clinical Epidemiology; Doi, S.A.R., Williamsk, G.M., Eds.; Springer: Berlin/Heidelberg, Germany, 2013.

40. Bolstad, G.; Benum, B.; Rokne, A. Anthropometry of Norwegian light industry and office workers. Appl. Ergon. 2001, 32, 239-246. [CrossRef]

41. Wang, M.J.; Wang, E.M.Y.; Lin, Y.C. The anthropometric database for children and young adults in Taiwan. Appl. Ergon. 2002, 33, 583-585. [CrossRef] 